\title{
Feeding the world, harmoniously
}

\author{
Tom Crompton
}

After-dinner debate about the long-term social and environmental impact of agricultural biotechnology on subsistence farming is often construed as a conversational indulgence of the well-fed. Indeed, in the course of its public relations struggles, the agribiotechnology industry itself has been keen to capitalize on the argument that the yield increases achievable through new biotechnologies present the only hope we have of continuing to feed a burgeoning world population.

To generalize, there is a marked distinction between the approach to agribiotechnology taken by regulators from more industrialized countries, who frequently advocate minimal regulatory intervention, and those from developing countries, who typically display a greater caution in the process of building a national biosafety framework, or negotiating international regulations. Biotechnology enthusiasts often attribute these differences of approach to the relative success of environmental lobbying groups in bending the ears of regulators in developing countries ${ }^{1}$. But it is in the developing world that the "feed the world" argument should be most compelling.

Can we really assume that governments in these countries, which readily subjugate environmental concern to economic pragmatism on many other issues, are just more easily duped by the scaremongery of environmental activists than their counterparts in the industrialized world? Some environmentalists may indeed be out of touch with the serious business of feeding hungry people, but doesn't it demonstrate an equal disregard for the needs of developing countries to dismiss governmental caution glibly, as reflecting nothing but a debilitating naiveness?

An adjunct to the argument forwarded by certain misguided agribiotechnology enthusiasts is that developing countries simply do not have the domestic know-how to formulate their own legislation, and must necessarily rely upon foreign expertise to assimilate existing regulations refined for use in industrialized countries. This assumption was implicit during the course of a biosafety workshop I recent-

Tom Crompton is at the Liaison Office of the International Center for Genetic Engineering and Biotechnology/United Nations International Development Organization, Vienna, and is a visiting fellow at the Department of Environmental Science, University of East London, Romford Road, London(crompton@uel.ac.uk). ly attended, where "experts" from industrialized countries were assembled on one side of the room, facing delegates from developing countries whom they were to advise on the form their national regulations should take.

\section{The interests of nations that will predominantly import genetically modified crops and those that will export them display important asymmetries.}

Many of these same experts-myself included-started out as molecular biologists who, back at the bench, would buoyantly assert the value-free nature of technology. "Science deals with facts," we'd say, as we washed our hands before leaving the lab for the day. "But when it comes to the economic or social impacts of technologies, the buck stops with political decision makers."

A thoroughgoing application of this philosophy among these same biologists in their new guises, as biosafety advisors to regulators from developing countries, would run something like this: "Here are the facts as we perceive them, and this is how our governments, given their particular agendas, have responded to them. How you respond will depend upon your assessment of your own local and national needs, which your boss may have been elected to address, and of which you will undoubtedly have a more intimate knowledge than we."

I was lambasted by representatives from industrialized countries at this same meeting for suggesting, within earshot of delegates from the developing world, that there are in fact real differences of interest between developing and industrialized countries with regard to agribiotechnology. Yet many developing country delegates themselves were aware of disparities of social and economic interest relating to such technologies.

The interests of nations that will predominantly import genetically modified crops and those that will export them display important asymmetries. Among the sources of these asymmetries are concerns that imported transgenic crops may not reflect the current needs of local farmers, worries about the economic impact of export substitution, the need of some companies based in temperate countries to overwinter lines in tropical climates before shipping seed back north for the growing season, and concerns about litigation in the event of a country encountering environmental problems with an imported crop. Consideration of such asymmetries should properly inform the continuing harmonization of national regulatory frameworks.

But recognition of each nation's unique regulatory interests is difficult to maintain under the constraints imposed by free-trade agreements. Indeed, the free-trade imperative is one of the main reasons that many governments and intergovernmental organizations advocate the harmonization of national regulatory frameworks. But even in the relatively homogeneous political and economic context of the European Union, individual member states have found the need for differing interpretations of the European Directive on Deliberate Release $(90 / 220 / \text { EEC) })^{2}$. How much greater a divergence in national legislation might be expected between developing and industrialized countries if regulatory frameworks were to reflect national priorities directly?

There is no doubt that harmonization of national legislation will facilitate international transfer of data on field releases, help avert possible national competition for more permissive environmental standards in order to attract foreign investment, and facilitate the development of new legislation in countries where not only is this currently lacking, but there are more pressing issues on the national agenda. And yet, regional trade agreements, investment preconditions laid down by transnational companies, government aid packages for capacity-building in biosafety, and reliance upon experts from industrialized countries can all tend to lead to the assimilation by developing countries of legislative frameworks that have been refined for use in North America or Europe ${ }^{2}$.

Regulatory harmony entails a need for consensus and therefore an overt consideration of points at which national interests diverge. All too often, however, such disparities are obfuscated by emotive recourse to those seductively simple arguments epitomized by the "feed the world" shibboleth.

\footnotetext{
1. Crompton, T. \& Tzotzos, G.T. J. Biolaw Business 2, 60-70 (1998).

2. R. von Schomberg, An Appraisal of the Working in Practice of Directive 90/220/EEC on the Deliberate Release of Genetically Modified Organisms: Working Document of the Scientific and Technological Options Assessment (STOA) (1998).
} 